\title{
From Reaction Models to Influence Graphs and Back: a Theorem ${ }^{\star}$
}

\author{
François Fages, Sylvain Soliman \\ Projet Contraintes, INRIA Rocquencourt, \\ BP105, 78153 Le Chesnay Cedex, France. \\ http://contraintes.inria.fr
}

\begin{abstract}
Biologists use diagrams to represent interactions between molecular species, and on the computer, diagrammatic notations are also more and more employed in interactive maps. These diagrams are fundamentally of two types: reaction graphs and activation/inhibition graphs. In this paper, we study the formal relationship between these graphs. We consider systems of biochemical reactions with kinetic expressions, as written in the Systems Biology Markup Language SBML, and interpreted by a system of Ordinary Differential Equations over molecular concentrations. We show that under a general condition of increasing monotonicity of the kinetic expressions, and in absence of both activation and inhibition effects between a pair of molecules, the influence graph inferred from the stoichiometric coefficients of the reactions is equal to the one defined by the signs of the coefficients of the Jacobian matrix. Under these conditions, satisfied by mass action law, Michaelis-Menten and Hill kinetics, the influence graph is thus independent of the precise kinetic expressions, and is computable in linear time in the number of reactions. We apply these results to Kohn's map of the mammalian cell cycle and to the MAPK signalling cascade. Then we propose a syntax for denoting antagonists in reaction rules and generalize our results to this setting.
\end{abstract}

\section{Introduction}

Biologists use diagrams to represent interactions between molecular species, and diagrammatic notations like the ones introduced by Kohn in his map of the mammalian cell cycle [2] are also employed on the computer in interactive maps, like for instance MIM ${ }^{1}$. This type of notation encompasses two types of information : interactions (binding, complexation, protein modification, etc.) and regulations (of an interaction or of a transcription).

The Systems Biology Markup Language (SBML) [3] uses a syntax of reaction rules with kinetic expressions to define reaction models in a precise way, and more

\footnotetext{
* This paper provides a direct presentation and a generalization of one theorem shown in [1] among other results in the framework of abstract interpretation which is not used here.

${ }^{1}$ http://discover.nci.nih.gov/mim/
} 
and more models are described in such a formalism, like in the biomodels.net repository. This type of language is well suited to describe interactions (and in a limited manner their regulations through the notion of modifiers) but not directly molecule to molecule activations and inhibitions.

On the other hand, formal influence graphs for activation and inhibition have been introduced in the setting of gene regulatory networks [4] as an abstraction of complex reaction networks. These graphs completely abstract from the precise interactions, especially at post-transcriptional level, and retain only the activation and inhibition effects between genes. In these influence graphs, the existence of a positive circuit (resp. a negative circuit) has been shown to be a necessary condition for multistationarity (resp. oscillations) in different settings [5-9], as conjectured by Thomas [10].

There are nowadays several tools providing different kinds of analyses for either reaction models or influence graphs. However the only formal relationship relating the two seems to be the extraction of the influence graph from the Jacobian matrix derived from the reaction model, when equipped with precise kinetic expressions and parameter values.

In this paper, we study more systematically the formal relationship between reaction models and activation/inhibition influence graphs. We consider systems of biochemical reactions with kinetic expressions, as written in the Systems Biology Markup Language SBML, and interpreted by systems of Ordinary Differential Equations over molecular concentrations. We show that under the general condition of strongly increasing monotonicity of the kinetic expressions, and in absence of both activation and inhibition effects from one molecule to the same target, the influence graph inferred from the stoichiometric coefficients of the reactions, called the syntactical influence graph, is identical to the influence graph defined by the signs of the coefficients of the Jacobian matrix, called the differential influence graph. Under these conditions, satisfied by mass action law, Michaelis-Menten and Hill kinetics, the influence graph is thus independent of the kinetic expressions for the reactions, and is computable in linear time in the number of reactions.

We show that this remarkable property applies to the transcription of Kohn's map of the mammalian cell cycle control [2] into an SBML model of approx. 800 reactions [11]. On this example, the syntactical influence graph is computed in less than one second, and our equivalence theorem shows that this influence graph would be the same as the differential influence graph for any standard kinetics and any (non zero) parameter values. The same property of independence from the kinetic expressions holds for the influence graph inferred from the MAPK signalling model of Levchenko et al. [12]. This influence graph exhibits positive as well as negative feedbacks that are hidden in the purely directional cascade of the reaction graph [13], and that have been the reason for an erroneous interpretation of Thomas' rules when applied to the MAPK cascade in $[14]$. 
Finally, we consider generalized reaction rules, where inhibitors can be indicated in the syntax of the rules, and generalize our results to this setting for a large set of kinetic expressions.

\section{Reaction Models}

Following SBML and BIOCHAM $[15,16]$ conventions, a model of a biochemical system is formally a set of reaction rules of the form $e$ for $S \Rightarrow S^{\prime}$ where $S$ is a set of molecules given with their stoichiometric coefficient, called a solution, $S^{\prime}$ is the transformed solution, and $e$ is a kinetic expression involving the concentrations of molecules (which are not strictly required to appear in $S$ ).

We will use the BIOCHAM operators + and $*$ to denote solutions as $2 * \mathrm{~A}$ $+B$, as well as the syntax of catalyzed reactions e for $S=[C] \Rightarrow S^{\prime}$ as an abbreviation for e for $\mathrm{S}+\mathrm{C}=\mathrm{S}^{\prime}+\mathrm{C}$.

Classical kinetic expressions are the mass action law kinetics

$$
k * \prod_{i=1}^{n} x_{i}^{l_{i}}
$$

for a reaction with $n$ reactants $x_{i}$, where $l_{i}$ is the stoichiometric coefficient of $x_{i}$ as a reactant, Michaelis-Menten kinetics

$$
V_{m} * x_{s} /\left(K_{m}+x_{s}\right)
$$

for an enzymatic reaction of the form $x_{s}=\left[x_{e}\right]=>x_{p}$, where ${ }^{2} V_{m}=k *\left(x_{e}+\right.$ $\left.x_{e} * x_{s} / K_{m}\right)$, and Hill's kinetics

$$
V_{m} * x_{s}^{n} /\left(K_{m}^{n}+x_{s}{ }^{n}\right)
$$

of which Michaelis-Menten kinetics is a special case with $n=1$.

A set of reaction rules $\left\{e_{i} \text { for } S_{i} \Rightarrow S_{i}^{\prime}\right\}_{i=1, \ldots, n}$ over molecular concentration variables $\left\{x_{1}, \ldots, x_{m}\right\}$, can be interpreted under different semantics. The traditional differential semantics interpret the rules by the following system of Ordinary Differential Equations (ODE):

$$
d x_{k} / d t=\sum_{i=1}^{n} r_{i}\left(x_{k}\right) * e_{i}-\sum_{j=1}^{n} l_{j}\left(x_{k}\right) * e_{j}
$$

where $r_{i}\left(x_{k}\right)$ (resp. $l_{i}$ ) is the stoichiometric coefficient of $x_{k}$ in the right (resp. left) member of rule $i$.

The differential semantics will be the only interpretation of reaction models considered here. In this paper, we shall not consider the other interpretations of

\footnotetext{
${ }^{2} x_{e} * x_{s} / K_{m}$ is the concentration of the enzyme-substrate complex, supposed constant in the Michaelian approximation and $x_{e}+x_{e} * x_{s} / K_{m}$ is thus the total amount of enzyme
} 
reaction rules used in BIOCHAM [1], namely the stochastic semantics, where the kinetic expressions are interpreted as transition probabilities, the rule set as a continuous-time Markov chain that can be simulated with Gillespie's algorithm [17], or the boolean semantics which simply forgets the kinetic expressions and interpret the rules as a non-deterministic (asynchronous) transition system over boolean states representing the absence or presence of molecules.

\section{Influence Graphs of Activation and Inhibition}

Influence graphs for activation and inhibition have been introduced for the analysis of gene expression in the setting of gene regulatory networks [4]. Such influence graphs are in fact an abstraction of complex reaction networks, and can be applied as such to protein interaction networks. However the distinction between the influence graph and the reaction (hyper)graph is crucial to the application of Thomas's conditions of multistationarity and oscillations $[4,7]$ to protein interaction network, and there has been some confusion between the two kinds of graphs [14].

Here we consider two definitions of the influence graph associated to a reaction model, and show their equivalence under general assumptions.

\subsection{Definition from the Jacobian Matrix}

In the differential semantics of a reaction rule model $M=\left\{e_{i}\right.$ for $l_{i} \Rightarrow r_{i} \mid i \in$ $I\}$ we have $\dot{x_{k}}=d x_{k} / d t=\sum_{i=1}^{n}\left(r_{i}\left(x_{k}\right)-l_{i}\left(x_{k}\right)\right) * e_{i}$. The Jacobian matrix $J$ is formed of the partial derivatives $J_{i j}=\partial \dot{x}_{i} / \partial x_{j}$.

Definition 1. The differential influence graph associated to a reaction model is the graph having for vertices the molecular species, and for edge-set the following two kinds of edges:

$\left\{A\right.$ activates $B \mid \partial \dot{x_{B}} / \partial x_{A}>0$ in some point of the space $\}$

$\cup\left\{A\right.$ inhibits $B \mid \partial \dot{x_{B}} / \partial x_{A}<0$ in some point of the space $\}$

Both activation and inhibition edges may exist between two molecular species in reaction models such as for instance:

$k_{1} * A$ for $A \Rightarrow B$

$k_{2} * A * B$ for $A+B \Rightarrow C$

We have indeed $d B / d t=k_{1} * A-k_{2} * A * B$ and $\partial \dot{B} / \partial A=k_{1}-k_{2} * B$, hence $A$ inhibits $B$ and $A$ activates $B$ both belong to the differential influence graph in such an example.

\subsection{Definition from the Stoichiometric Coefficients}

Definition 2. The syntactical influence graph associated to a reaction model $M$ is the graph having for vertices the molecular species, and for edges the following set: 


$$
\begin{array}{ll}
\{A \text { inhibits } B \quad & \mid \exists\left(e_{i} \text { for } l_{i}=>r_{i}\right) \in M \\
& \left.l_{i}(A)>0 \text { and } r_{i}(B)-l_{i}(B)<0\right\} \\
\cup\{A \text { activates } B & \mid \exists\left(e_{i} \text { for } l_{i}=>r_{i}\right) \in M, \\
& \left.l_{i}(A)>0 \text { and } r_{i}(B)-l_{i}(B)>0\right\}
\end{array}
$$

In particular, we have the following influences for elementary reactions of complexation, modification, synthesis and degradation:

$\alpha(\{\mathrm{A}+\mathrm{B}=>\mathrm{C}\})=\{\mathrm{A}$ inhibits $\mathrm{B}, \mathrm{A}$ inhibits $\mathrm{A}, \mathrm{B}$ inhibits $\mathrm{A}$,

$\mathrm{B}$ inhibits $\mathrm{B}, \mathrm{A}$ activates $\mathrm{C}, \mathrm{B}$ activates $\mathrm{C}\}$

$\alpha(\{\mathrm{A}=[\mathrm{C}]=>\mathrm{B}\})=\{\mathrm{C}$ inhibits $\mathrm{A}, \mathrm{A}$ inhibits $\mathrm{A}, \mathrm{A}$ activates $\mathrm{B}, \mathrm{C}$ activates $\mathrm{B}\}$

$\alpha\left(\left\{\mathrm{A}=[\mathrm{B}]=>_{-}\right\}\right)=\{\mathrm{B}$ inhibits $\mathrm{A}, \mathrm{A}$ inhibits $\mathrm{A}\}$

$\alpha\left(\left\{{ }_{-}=[\mathrm{B}]=>\mathrm{A}\right\}\right)=\{\mathrm{B}$ activates $\mathrm{A}\}$

The inhibition loops on the reactants are justified by the negative sign in the Jacobian matrix of the differential semantics of such reactions. Unlike the differential influence graph, this graph is clearly trivial to compute by browsing the syntax of the rules:

Proposition 1. The syntactical influence graph of a reaction model of $n$ rules is computable in $O(n)$ time.

\subsection{Over-approximation Theorem}

Comparing the differential influence graph and the syntactical influence graph requires that the information in the kinetic expressions and in the reactions be compatible. This motivates the following definition where the first property forbids the presence of purely kinetic inhibitors not represented in the reaction, and the second property enforces that the variables appearing in the kinetic expressions do appear as reactants or enzymes in the reaction.

Definition 3. In a reaction rule $e$ for $l=>r$, we say that a kinetic expression $e$ is increasing iff for all molecules $x_{k}$ we have

1. $\partial e / \partial x_{k} \geq 0$ in all points of the space,

2. $l\left(x_{k}\right)>0$ if $\partial e / \partial x_{k}>0$ in some point of the space.

$A$ reaction model has an increasing kinetics iff all its reaction rules have an increasing kinetics.

One can easily check that:

Proposition 2. Mass action law kinetics for any reaction, as well as Michaelis Menten and Hill kinetics for enzymatic reactions, are increasing.

On the other hand, negative Hill kinetics of the form $k_{1} /\left(k_{2}^{n}+y^{n}\right)$ are not increasing. They represent an inhibition by a molecule $y$ not belonging to the reactants, and thus not reflected in the syntax of the reaction.

Theorem 1. For any reaction model with an increasing kinetics, the differential influence graph is a subgraph of the syntactical influence graph. 
Proof. If ( $A$ activates $B$ ) belongs to the differential influence graph then $\partial \dot{B} / \partial A>$ 0 . Hence there exists a term in the differential equation for $B$, of the form $\left(r_{i}(B)-l_{i}(B)\right) * e_{i}$ with $\partial e_{i} / \partial A$ of the same sign as $r_{i}(B)-l_{i}(B)$.

Let us suppose that $r_{i}(B)-l_{i}(B)>0$ then $\partial e_{i} / \partial A>0$ and since $e_{i}$ is increasing we get that $l_{i}(A)>0$ and thus that $(A$ activates $B)$ in the syntactical graph. If on the contrary $r_{i}(B)-l_{i}(B)<0$ then $\partial e_{i} / \partial A<0$, which is not possible for an increasing kinetics.

If $(A$ inhibits $B)$ is in the differential graph then $\partial \dot{B} / \partial A<0$. Hence there exists a term in the differential semantics, of the form $\left(r_{i}(B)-l_{i}(B)\right) * e_{i}$ with $\partial e_{i} / \partial A$ of sign opposite to that of $r_{i}(B)-l_{i}(B)$.

Let us suppose that $r_{i}(B)-l_{i}(B)>0$ then $\partial e_{i} / \partial A<0$, which is not possible for an increasing kinetics. If on the contrary $r_{i}(B)-l_{i}(B)<0$ then $\partial e_{i} / \partial A>0$ and since $e_{i}$ is increasing we get that $l_{i}(A)>0$ and thus that $(A$ activates $B$ ) is in the syntactical influence graph.

Corollary 1. For any reaction model with an increasing kinetics, the differential influence graph restricted to the phase space w.r.t. some initial conditions, is a subgraph of the syntactical influence graph.

Proof. Restricting the points of the phase space to those points that are accessible from some initial states, restricts the number of edges in the differential influence graphs which thus remains a subgraph of the syntactical influence graph.

It is worth noticing that even in the simple case of mass action law kinetics, the differential influence graph may be a strict subset of the syntactical influence graph. For instance let $x$ be the following model :

$$
\begin{aligned}
& k_{1} * A \text { for } A=>B \\
& k_{2} * A \text { for }-=[A]=>A
\end{aligned}
$$

In the syntactical influence graph, $A$ activates $B, A$ activates $A$ and $A$ inhibits $A$, however $\dot{A}=\left(k_{2}-k_{1}\right) * A$, hence $\partial \dot{A} / \partial A$ can be made always positive or always negative or always null, resulting in the absence of respectively, $A$ inhibits $A, A$ activates $A$ or both, in the differential influence graph.

\subsection{Equivalence Theorem}

Definition 4. In a reaction rule $e$ for $l=>r$, a kinetic expression $e$ is strongly increasing iff for all molecules $x_{k}$ we have

1. $\partial e / \partial x_{k} \geq 0$ in all points of the space,

2. $l\left(x_{k}\right)>0$ if and only if there exists a point in the space s.t. $\partial e / \partial x_{k}>0$

A reaction model has a strongly increasing kinetics iff all its reaction rules have a strongly increasing kinetics.

Note that strongly increasing implies increasing.

Proposition 3. Mass action law kinetics for any reaction, as well as Michaelis Menten and Hill kinetics for enzymatic reactions, are strongly increasing. 
Proof. For the case of Mass action law, the kinetics are of the form:

$$
e_{i}=k_{i} * \prod_{l=1}^{m} x_{l}^{l_{i}\left(x_{l}\right)}
$$

with $k_{i}>0$ and $l_{i}\left(x_{l}\right) \geq 0$. We thus have $\partial e_{i} / \partial x_{k}=0$ if $l_{i}\left(x_{k}\right)=0$ and $\partial e_{i} / \partial x_{k}=k_{i} * l_{i}\left(x_{k}\right) * x_{k}^{l_{i}\left(x_{k}\right)-1} \prod_{l \neq k} x_{l}^{l_{i}\left(x_{l}\right)}$ otherwise, which clearly satisfies (1) and $(2)$.

In the case of Hill kinetics (of which Michaelis Menten is a subcase), we have:

$$
e_{i}=\frac{V_{m} * x_{s}^{n}}{K_{m}^{n}+x_{s}^{n}}
$$

for the reaction $x_{s}+x_{e}=>x_{p}+x_{e}$ and where $V_{m}=k_{2} * x_{e}^{t o t}=k_{2} *\left(x_{e}+\right.$ $\left.k_{1} * x_{e} * x_{s} /\left(k_{-1}+k_{2}\right)\right)$ from the steady state approximation. It is obvious that $\partial e_{i} / \partial x_{k}=0$ for all $x_{k}$ other than $x_{s}$ and $x_{e}$ since they do not appear in $e_{i}$ and one can easily check that with all the constants $n, k_{1}, k_{-1}, k_{2}$ strictly positive, both $\partial e_{i} / \partial x_{e}$ and $\partial e_{i} / \partial x_{s}$ are greater than 0 at some point in the space.

Lemma 1. Let $M$ be a reaction model with a strongly increasing kinetics,

If $(A$ activates $B)$ is an edge in the syntactical influence graph, and not ( $A$ inhibits $B)$, then ( $A$ activates $B$ ) belongs to the differential influence graph.

If $(A$ inhibits $B)$ is an edge in the syntactical influence graph, and not ( $A$ activates $B$ ), then (A inhibits $B$ ) belongs to the differential influence graph.

Proof. Since $\partial \dot{B} / \partial A=\sum_{i=1}^{n}\left(r_{i}(B)-l_{i}(B)\right) * \partial e_{i} / \partial A$ and all $e_{i}$ are increasing we get that $\partial \dot{B} / \partial A=\sum_{\left\{i \leq n \mid l_{i}(A)>0\right\}}\left(r_{i}(B)-l_{i}(B)\right) * \partial e_{i} / \partial A$.

Now if $(A$ activates $B)$ is in the syntactical influence graph, but not $(A$ inhibits $B$ ), then all rules such that $l_{i}(A)>0$ verify $r_{i}(B)-l_{i}(B) \geq 0$ and there is at least one rule for which the inequality is strict. We thus get that $\partial \dot{B} / \partial A$ is a sum of positive numbers, amongst which one is such that $r_{i}(B)-l_{i}(B)>0$ and $l_{i}(A)>0$ which, since $M$ is strongly increasing, implies that there exists a point in the space for which $\partial e_{i} / \partial A>0$. Hence $\partial \dot{B} / \partial A>0$ at that point, and ( $A$ activates $B$ ) is thus in the differential influence graph.

For inhibition the same reasoning applies with the opposite sign for the $r_{i}(B)-l_{i}(B)$ and thus for the partial derivative $\partial \dot{B} / \partial A$.

This lemma establishes the following equivalence result:

Theorem 2. In a reaction model with a strongly increasing kinetics and where no molecule is at the same time an activator and an inhibitor of the same target molecule, the differential and syntactical influence graphs coincide.

This theorem shows that for standard kinetic expressions, the syntactical influences coincide with the differential influences based on the signs of the coefficients in the Jacobian matrix, when no molecule is at the same time an activator and an inhibitor of the same molecule. The theorem thus provides a linear time algorithm for computing the differential influences in these cases, simply by computing the syntactical influences. It shows also that the differential influence graph is independent of the kinetic expressions. 
Corollary 2. The differential influence graph of a reaction model of $n$ rules with a strongly increasing kinetics is computable in time $O(n)$ if no molecule is at the same time an activator and an inhibitor.

Corollary 3. The differential influence graph of a reaction model is independent of the kinetic expressions as long as they are strongly increasing, if no molecule is at the same time an activator and an inhibitor.

\section{Application to Kohn's Map of the Mammalian Cell Cycle Control}

Kohn's map of the mammalian cell cycle control [2] has been transcribed in BIOCHAM to serve as a large benchmarking example of approx. 500 species and 800 rules [11].

On Kohn's map, the computation of activation and inhibition influences takes less than one second $\mathrm{CPU}$ time (on a PC 1,7GHz) for the complete model, showing the efficiency of the syntactical inference algorithm. The influence graph is composed of 1231 activation edges and 1089 inhibition edges.

Furthermore in this large example no molecule is both an activator and an inhibitor of the same target molecule. Theorem 2 thus entails that the computed influence graph is equal to the differential graph that would be obtained in any kinetic model of Kohn's map for any standard kinetic expressions and for any (non zero) parameter values.

Since there is a lot of kinetic data missing for such a big model, the possibility to nevertheless obtain the exact influence graph without having to estimate parameters or even to choose precise kinetic expressions is quite remarkable, and justifies the use of purely qualitative models for the analysis of feedback circuits.

\section{Application to the Signal Transduction MAPK "cascade"}

Let us consider the MAPK signalling model of [12]. Figure 1 depicts the reaction graph as a bipartite graph with round boxes for molecules and rectangular boxes for rules. Figure 2 depicts the syntactical influence graph, where activation (resp. inhibition) is materialized by plain (resp. dashed) arrows.

This computed graph reveals the negative feedbacks that are somewhat hidden in a purely directional signalling cascade of reactions. Furthermore, as no molecule is at the same time an activator and an inhibitor of a same molecule, this graph is largely independent of the kinetics of the reactions, and would be identical to the differential influence graph for any standard kinetic expressions with any (non zero) kinetic parameter values.

These negative feedbacks, a necessary condition for oscillations $[4,8,9]$, have been formally analyzed in [13] and interpreted as enzyme sequestration in complexes. Furthermore, oscillations in the MAPK cascade model have been shown in $[18]$. 


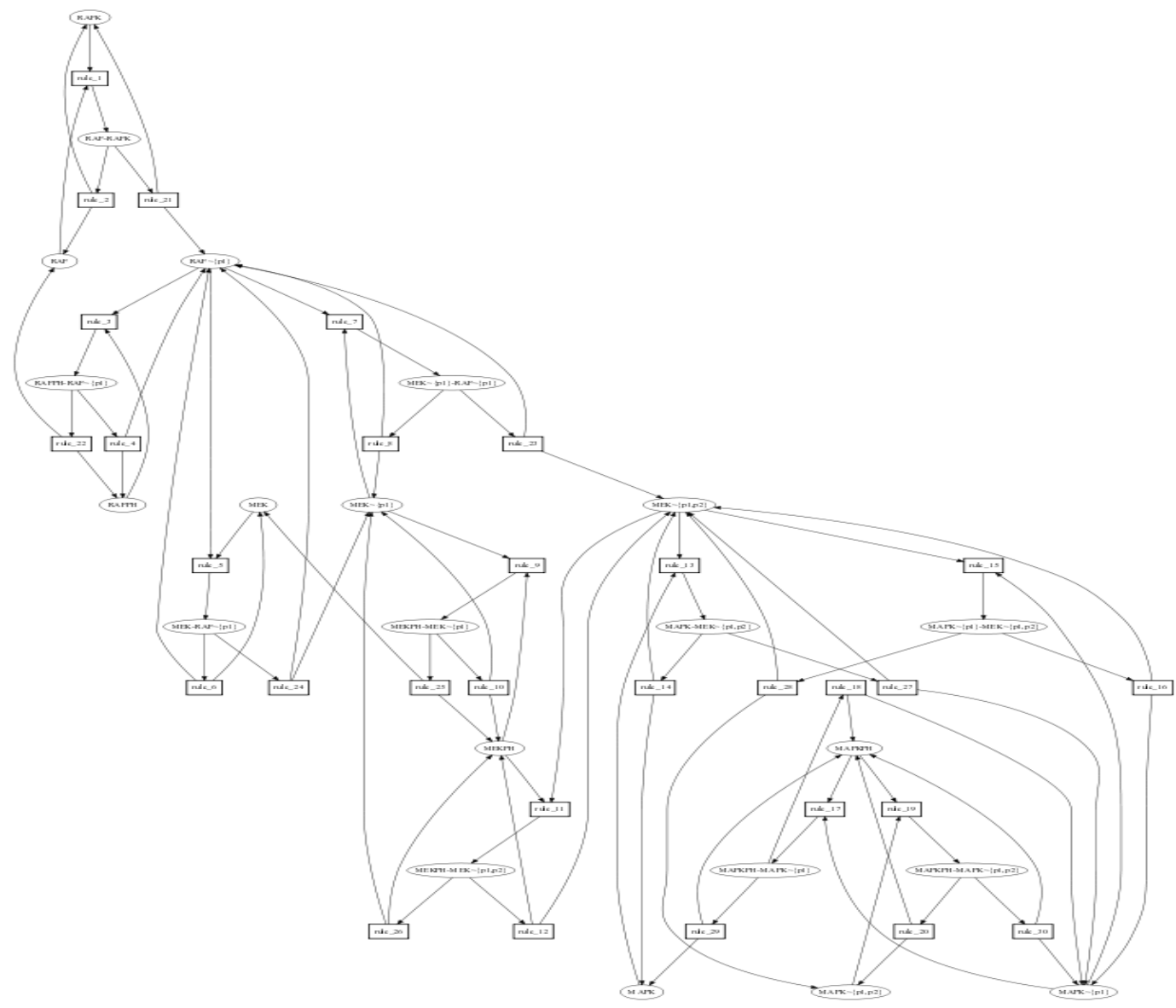

Fig. 1. Reaction graph of the MAPK model of[12].

The influence graph also exhibits positive circuits. These are a necessary condition for multistationarity $[4,7]$ that has been observed in the MAPK model, and experimentally in Xenopus oocytes [14]. Note that the absence of circuit in the (directional) reaction graph of MAPK was misinterpreted as a counterexample to Thomas' rule in [14] because of a confusion between both kinds of graphs.

\section{Adding a Syntax for Antagonists in Reaction Rules}

The over-approximation theorem 1 may suggest to provide a syntax for antagonists (i.e. inhibitors) in reaction rules, and generalize the result to this setting. Note that the mixing of mechanistic reaction models with non-mechanistic 


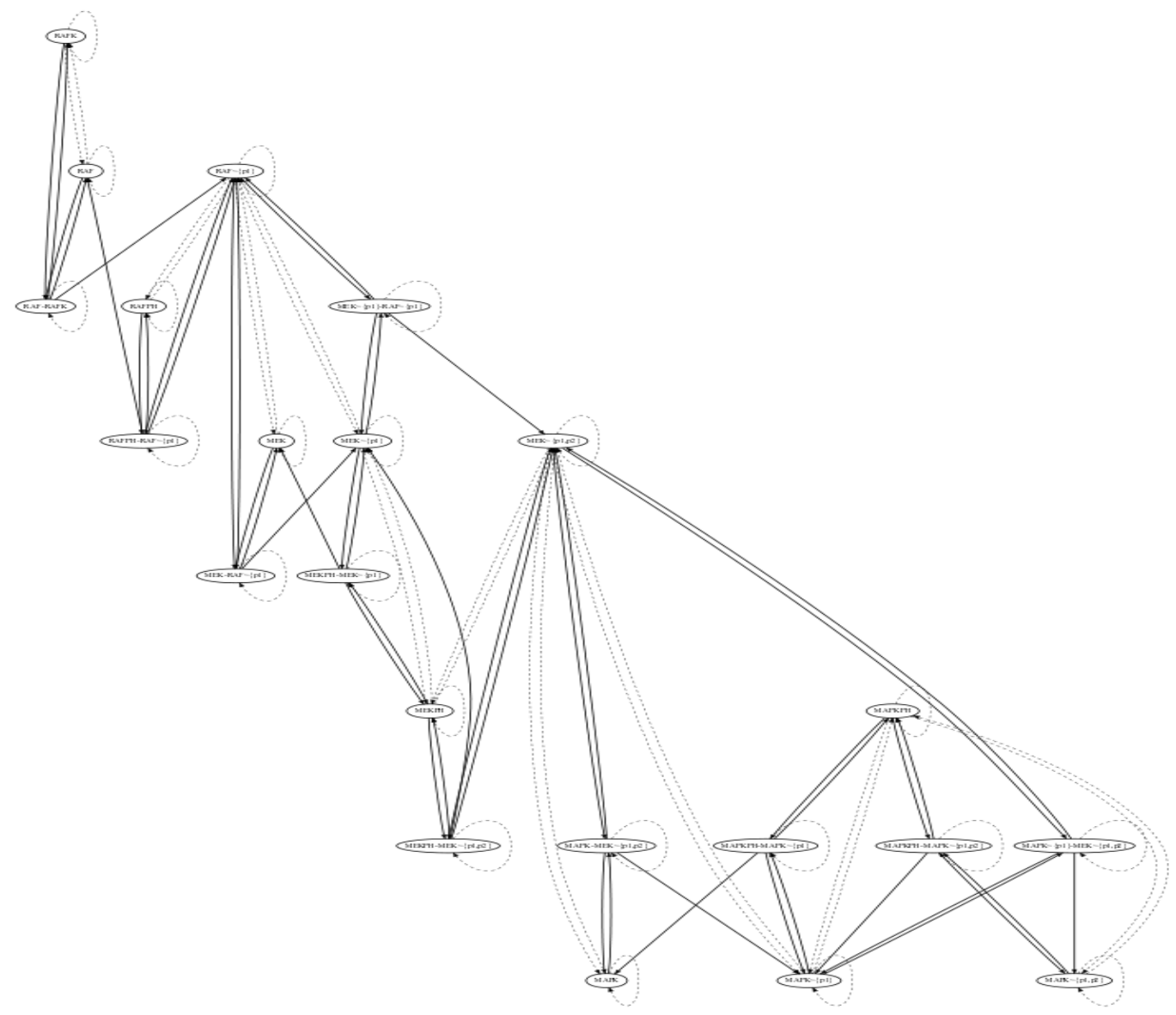

Fig. 2. Influence graph inferred from the MAPK reaction model.

information on the inhibitors of some reactions, is a common practice in diagrammatic notations which often combine reaction edges with activation and inhibition edges.

Let us denote by ( $e$ for $l=[/ a] \Rightarrow r$ ) a generalized reaction rule with antagonists $a$. Reaction rules with catalysts, of the form ( $e$ for $l=[c / a] \Rightarrow r$ ), will remain an abbreviation for ( $e$ for $l+c=[/ a]=>r+c$ ). This notation for antagonists thus provides a counterpart for denoting the inhibitory effect of some agent on a reaction, symmetrically to the activation effect of the catalysts of the reaction.

Definition 5. The syntactical influence graph associated to a generalized reaction model $M$ is the graph having for vertices the molecular species, and for edges the following set: 


$$
\begin{array}{c|l}
\{A \text { inhibits } B & \mid \exists\left(e_{i} \text { for } l_{i}=\left[/ a_{i}\right]=>r_{i}\right) \in M, \\
& \left.l_{i}(A)>0 \text { and } r_{i}(B)-l_{i}(B)<0\right\} \\
\cup\{A \text { activates } B & \mid \exists\left(e_{i} \text { for } l_{i}=\left[/ a_{i}\right]=>r_{i}\right) \in M, \\
& \left.l_{i}(A)>0 \text { and } r_{i}(B)-l_{i}(B)>0\right\} \\
\cup\{A \text { activates } B & \mid \exists\left(e_{i} \text { for } l_{i}=\left[/ a_{i}\right]=>r_{i}\right) \in M, \\
& \left.a_{i}(A)>0 \text { and } r_{i}(B)-l_{i}(B)<0\right\} \\
\cup\{A \text { inhibits } B \quad & \mid \exists\left(e_{i} \text { for } l_{i}=\left[/ a_{i}\right]=>r_{i}\right) \in M, \\
& \left.a_{i}(A)>0 \text { and } r_{i}(B)-l_{i}(B)>0\right\}
\end{array}
$$

For instance, the set of syntactical influences of the generalized reaction rule $\mathrm{A}=[/ \mathrm{I}] \Rightarrow \mathrm{B}\}$ is $\{A$ inhibits $A, I$ activates $A, A$ activates $B, I$ inhibits $B\}$. On the other hand, note that the definition of the differential influence graph applies to generalized reaction models as it is based on the kinetic expressions only.

Definition 6. In a generalized reaction rule $e$ for $l=[/ a]=>r$, a kinetic expression e is compatible iff for all molecules $x_{k}$ we have

1. $l\left(x_{k}\right)>0$ if there exists a point in the space s.t. $\partial e / \partial x_{k}>0$,

2. $a\left(x_{k}\right)>0$ if there exists a point in the space s.t. $\partial e / \partial x_{k}<0$.

A generalized reaction model has a compatible kinetics iff all its reaction rules have a compatible kinetics.

For instance, a kinetics of the form $\mathrm{k} 1 * \mathrm{Mdm} 2 /(\mathrm{k} 2+\mathrm{P} 53)$ for the generalized reaction rule Mdm2 $=[/ \mathrm{P} 53] \Rightarrow$ Mdm2p expressing the phosphorylation of Mdm2 that is inhibited by P53 (see [19]) is compatible.

Note that for a reaction model, strongly increasing implies compatible. Furthermore, we have:

Theorem 3. For any generalized reaction model with a compatible kinetics, the differential influence graph is a subgraph of the syntactical influence graph.

Proof. If ( $A$ activates $B$ ) belongs to the differential influence graph then $\partial \dot{B} / \partial A>$ 0 . Hence there exists a term in the differential equation for $B$, of the form $\left(r_{i}(B)-l_{i}(B)\right) * e_{i}$ with $\partial e_{i} / \partial A$ of the same sign as $r_{i}(B)-l_{i}(B)$.

Let us suppose that $r_{i}(B)-l_{i}(B)>0$ then $\partial e_{i} / \partial A>0$, and since $e_{i}$ is compatible we get that $l_{i}(A)>0$ and thus that $(A$ activates $B)$ in the syntactical graph. If on the contrary $r_{i}(B)-l_{i}(B)<0$ then $\partial e_{i} / \partial A<0$, and since $e_{i}$ is compatible we get that $a_{i}(A)>0$ and thus that $(A$ activates $B)$ is in the syntactical influence graph.

If $(A$ inhibits $B)$ is in the differential graph then $\partial \dot{B} / \partial A<0$. Hence there exists a term in the differential semantics, of the form $\left(r_{i}(B)-l_{i}(B)\right) * e_{i}$ with $\partial e_{i} / \partial A$ of sign opposite to that of $r_{i}(B)-l_{i}(B)$.

Let us suppose that $r_{i}(B)-l_{i}(B)>0$ then $\partial e_{i} / \partial A<0$, and since $e_{i}$ is compatible we get that $a_{i}(A)>0$ and thus that $(A$ inhibits $B)$ is in the syntactical influence graph. If on the contrary $r_{i}(B)-l_{i}(B)<0$ then $\partial e_{i} / \partial A>0$, and since $e_{i}$ is compatible we get that $l_{i}(A)>0$ and thus that $(A$ activates $B)$ is in the syntactical influence graph. 
This theorem shows that in this setting which mixes reaction rules with information on antagonists, the syntactical influence graph still over-approximates the differential influence graph for any standard kinetics.

\section{Conclusion}

This work shows that to a large extent, the influence graph of a reaction model is independent of the kinetic parameters and kinetic expressions, and that it can be computed in linear time simply from the syntax of the reactions. This happens for strongly increasing kinetics such as classical mass action law, Michaelis-Menten and Hill kinetics, when no molecule is at the same time an activator and an inhibitor of a same target molecule.

The inference of the syntactical influence graph from a reaction model has been implemented in BIOCHAM, and applied to various models. On a transcription of Kohn's map into approx. 800 reaction rules, this implementation shows that no molecule is at the same time an activator and an inhibitor of a same molecule, and therefore, our equivalence theorem states that the differential influence graph would be the same for any standard kinetics with any parameter values.

On the MAPK signalling cascade that does not contain any feedback reaction, the implementation does reveal both positive and negative feedback circuits in the influence graph, which has been a source of confusion for the correct application of Thomas' rules. Furthermore, in this example again, no molecule is at the same time an activator and an inhibitor of another molecule, showing the independence of the influence graph from the kinetics.

Acknowledgement. This work benefited from partial support of the European Union FP6 Network of Excellence REWERSE http://www.rewerse.net, and Strep TEMPO http://www. chrono-tempo.org.

\section{References}

1. Fages, F., Soliman, S.: Abstract interpretation and types for systems biology. To appear in Theoretical Computer Science (2008)

2. Kohn, K.W.: Molecular interaction map of the mammalian cell cycle control and DNA repair systems. Molecular Biology of the Cell 10 (1999) 2703-2734

3. Hucka, M., et al.: The systems biology markup language (SBML): A medium for representation and exchange of biochemical network models. Bioinformatics $\mathbf{1 9}$ (2003) 524-531

4. Thomas, R., Gathoye, A.M., Lambert, L.: A complex control circuit : regulation of immunity in temperate bacteriophages. European Journal of Biochemistry 71 (1976) 211-227

5. Kaufman, M., Soulé, C., Thomas, R.: A new necessary condition on interaction graphs for multistationarity. Journal of Theoretical Biology 248 (2007) 675-685

6. Soulé, C.: Mathematical approaches to differentiation and gene regulation. C. R. Biologies 329 (2006) 13-20 
7. Soulé, C.: Graphic requirements for multistationarity. ComplexUs 1 (2003) 123133

8. Snoussi, E.: Necessary conditions for multistationarity and stable periodicity. J. Biol. Syst. 6 (1998) 3-9

9. Gouzé, J.L.: Positive and negative circuits in dynamical systems. J. Biol. Syst. 6 (1998) 11-15

10. Thomas, R.: On the relation between the logical structure of systems and their ability to generate multiple steady states or sustained oscillations. Springer Ser. Synergetics 9 (1981) 180-193

11. Chabrier-Rivier, N., Chiaverini, M., Danos, V., Fages, F., Schächter, V.: Modeling and querying biochemical interaction networks. Theoretical Computer Science $\mathbf{3 2 5}$ (2004) 25-44

12. Levchenko, A., Bruck, J., Sternberg, P.W.: Scaffold proteins may biphasically affect the levels of mitogen-activated protein kinase signaling and reduce its threshold properties. PNAS 97 (2000) 5818-5823

13. Ventura, A.C., Sepulchre, J.A., Merajver, S.D.: A hidden feedback in signaling cascades is revealed. PLoS Computational Biology to appear (2008)

14. Markevich, N.I., Hoek, J.B., Kholodenko, B.N.: Signaling switches and bistability arising from multisite phosphorylation in protein kinase cascades. Journal of Cell Biology 164 (2005) 353-359

15. Calzone, L., Fages, F., Soliman, S.: BIOCHAM: An environment for modeling biological systems and formalizing experimental knowledge. BioInformatics 22 (2006) 1805-1807

16. Fages, F., Soliman, S., Chabrier-Rivier, N.: Modelling and querying interaction networks in the biochemical abstract machine BIOCHAM. Journal of Biological Physics and Chemistry 4 (2004) 64-73

17. Gillespie, D.T.: Exact stochastic simulation of coupled chemical reactions. Journal of Physical Chemistry 81 (1977) 2340-2361

18. Qiao, L., Nachbar, R.B., Kevrekidis, I.G., Shvartsman, S.Y.: Bistability and oscillations in the huang-ferrell model of mapk signaling. PLoS Computational Biology 3 (2007) 1819-1826

19. Ciliberto, A., Novák, B., Tyson, J.J.: Steady states and oscillations in the p53/mdm2 network. Cell Cycle 4 (2005) 488-493 